J. Korean Math. Soc. 49 (2012), No. 6, pp. 1111-1121

http://dx.doi.org/10.4134/JKMS.2012.49.6.1111

\title{
TETRAVALENT SYMMETRIC GRAPHS OF ORDER $9 p$
}

\author{
Song-Tao Guo and Yan-Quan FenG
}

ABstract. A graph is symmetric if its automorphism group acts transitively on the set of arcs of the graph. In this paper, we classify tetravalent symmetric graphs of order $9 p$ for each prime $p$.

\section{Introduction}

Let $G$ be a permutation group on a set $\Omega$ and $\alpha \in \Omega$. Denote by $G_{\alpha}$ the stabilizer of $\alpha$ in $G$, that is, the subgroup of $G$ fixing the point $\alpha$. We say that $G$ is semiregular on $\Omega$ if $G_{\alpha}=1$ for every $\alpha \in \Omega$ and regular if $G$ is transitive and semiregular. Throughout this paper, we consider undirected finite connected graphs without loops or multiple edges. For a graph $X$ we use $V(X), E(X)$ and $\operatorname{Aut}(X)$ to denote its vertex set, edge set, and automorphism group, respectively. For $u, v \in V(X)$, denote by $\{u, v\}$ the edge incident to $u$ and $v$ in $X$.

A graph $X$ is said to be vertex-transitive if $\operatorname{Aut}(X)$ acts transitively on $V(X)$. An $s$-arc in a graph is an ordered $(s+1)$-tuple $\left(v_{0}, v_{1}, \ldots, v_{s-1}, v_{s}\right)$ of vertices of the graph $X$ such that $v_{i-1}$ is adjacent to $v_{i}$ for $1 \leq i \leq s$, and $v_{i-1} \neq v_{i+1}$ for $1 \leq i \leq s-1$. In particular, a 1-arc is called an arc for short and a 0 -arc is a vertex. For a subgroup $G \leq \operatorname{Aut}(X)$, a graph $X$ is said to be $(G, s)$-arctransitive and $(G, s)$-regular if $G$ is transitive and regular on the set of $s$-arcs in $X$, respectively. A $(G, s)$-arc-transitive graph is said to be $(G, s)$-transitive if it is not $(G, s+1)$-arc-transitive. In particular, a $(G, 1)$-arc-transitive graph is simply called $G$-symmetric. A graph $X$ is simply called s-arc-transitive, sregular and $s$-transitive if it is $(\operatorname{Aut}(X), s)$-arc-transitive, $(\operatorname{Aut}(X), s)$-regular and $(\operatorname{Aut}(X), s)$-transitive, respectively.

Arc-transitive or $s$-transitive graphs have received considerable attention in the literature. For example, $s$-transitive graphs of order $n p$ was classified in $[3,4,23]$ depending on $n=1,2$ or 3 , where $p$ is a prime. $\mathrm{Li}$ [13] showed that there exists an $s$-transitive graph of odd order if and only if $s \leq 3$. For the case of valency 4, Gardiner and Praeger $[8,9]$ characterized tetravalent

Received May 7, 2010.

2010 Mathematics Subject Classification. 05C25, $20 \mathrm{~B} 25$.

Key words and phrases. normal Cayley graph, symmetric graph, s-transitive graph. 
symmetric graphs, and $\mathrm{Li}$ et al. [14] classified vertex-primitive tetravalent $s$ transitive graphs. The classification of tetravalent $s$-transitive Cayley graphs on abelian groups was given by $\mathrm{Xu}$ and $\mathrm{Xu}[25]$. We may deduce a classification of tetravalent 1-regular Cayley graphs on dihedral groups from [12, 18, 21, 22]. Zhou [31] gave a classification of tetravalent 1-regular graphs of order $2 p q$ for $p, q$ primes. Recently, Zhou [29] classified tetravalent $s$-transitive graphs of order $4 p$, and Zhou and Feng [30] classified tetravalent $s$-transitive graphs of order $2 p^{2}$. In this paper we classify tetravalent $s$-transitive graphs of order $9 p$.

Throughout the paper we denote by $C_{n}$ and $K_{n}$ the cycle and the complete graph of order $n$, respectively. Denote by $\mathbb{Z}_{n}$ the cyclic group of order $n$, by $\mathbb{Z}_{n}^{*}$ the multiplicative group of $\mathbb{Z}_{n}$ consisting of numbers coprime to $n$, by $D_{2 n}$ the dihedral group of order $2 n$, and by $F_{n}$ the Frobenius group of order $n$.

\section{Preliminary results}

For a subgroup $H$ of a group $G$, denote by $C_{G}(H)$ the centralizer of $H$ in $G$ and by $N_{G}(H)$ the normalizer of $H$ in $G$.

Proposition 2.1 ([11, Chapter I, Theorem 4.5]). The quotient group

$$
N_{G}(H) / C_{G}(H)
$$

is isomorphic to a subgroup of the automorphism group $\operatorname{Aut}(H)$ of $H$.

The following proposition is due to Burnside.

Proposition 2.2 ([19, Theorem 8.5.3]). Let $p$ and $q$ be primes, and let $m$ and $n$ be non-negative integers. Then every group of order $p^{m} q^{n}$ is solvable.

Let $G$ be a permutation group on a set $\Omega$. The size of $\Omega$ is called the degree of $G$ acting on $\Omega$.

Proposition 2.3 ([6, Corollary 3.5B]). Every transitive permutation group of prime degree $p$ is either 2-transitive or solvable with a regular normal Sylow p-subgroup.

The following proposition is about the permutation group of degree $p^{2}$ for $p$ a prime.

Proposition 2.4 ([28, Proposition 1]). Any transitive group of degree $p^{2}$ has a regular subgroup.

For a finite group $G$ and a subset $S$ of $G$ such that $1 \notin S$ and $S=S^{-1}$, the Cayley graph $\operatorname{Cay}(G, S)$ on $G$ with respect to $S$ is defined to have vertex set $V(\operatorname{Cay}(G, S))=G$ and edge set $E(\operatorname{Cay}(G, S))=\{\{g, s g\} \mid g \in G, s \in S\}$. Clearly, a Cayley graph $\operatorname{Cay}(G, S)$ is connected if and only if $S$ generates $G$. Furthermore, $\operatorname{Aut}(G, S)=\left\{\alpha \in \operatorname{Aut}(G) \mid S^{\alpha}=S\right\}$ is a subgroup of the automorphism group $\operatorname{Aut}(\operatorname{Cay}(G, S))$. Given a $g \in G$, define the permutation $R(g)$ on $G$ by $x \mapsto x g, x \in G$. Then $R(G)=\{R(g) \mid g \in G\}$, called the right regular representation of $G$, is a permutation group isomorphic to $G$. The 
Cayley graph is vertex-transitive because it admits the right regular representation $R(G)$ of $G$ as a regular group of automorphisms of $\operatorname{Cay}(G, S)$. A Cayley graph $\operatorname{Cay}(G, S)$ is said to be normal if $R(G)$ is normal in $\operatorname{Aut}(\operatorname{Cay}(G, S))$. A graph $X$ is isomorphic to a Cayley graph on $G$ if and only if $\operatorname{Aut}(X)$ has a subgroup isomorphic to $G$, acting regularly on vertices (see [20]). For two subsets $S$ and $T$ of $G$ not containing the identity 1 , if there is an $\alpha \in \operatorname{Aut}(G)$ such that $S^{\alpha}=T$, then $S$ and $T$ are said to be equivalent, denoted by $S \equiv T$. We may easily show that if $S \equiv T$, then $\operatorname{Cay}(G, S) \cong \operatorname{Cay}(G, T)$ and $\operatorname{Cay}(G, S)$ is normal if and only if $\operatorname{Cay}(G, T)$ is normal.

Proposition 2.5 ([26, Proposition 1.5]). A Cayley graph $\mathrm{Cay}(G, S)$ is normal if and only if $\operatorname{Aut}(\operatorname{Cay}(G, S))_{1}=\operatorname{Aut}(G, S)$, where $\operatorname{Aut}(\operatorname{Cay}(G, S))_{1}$ is the stabilizer of 1 in $\operatorname{Aut}(\operatorname{Cay}(G, S))$.

From [1, Corollary 1.3], we have the following proposition.

Proposition 2.6. Let $X=\operatorname{Cay}(G, S)$ be a connected tetravalent Cayley graph on a finite abelian group $G$ of odd order. Then $X$ is normal except for $G=\mathbb{Z}_{5}$ and $X=K_{5}$.

For two subgroups $M$ and $N$ of a group $G, M \rtimes N$ stands for the semidirect product of $M$ by $N$. The next proposition characterizes the vertex stabilizers of connected tetravalent $s$-transitive graphs (see [14, Lemma 2.5] and [13, Theorem 1.1]).

Proposition 2.7. Let $X$ be a connected tetravalent $(G, s)$-transitive graph of odd order. Then $s \leq 3$ and the stabilizer $G_{v}$ of a vertex $v \in V(X)$ in $G$ is as follows:

(1) $G_{v}$ is a 2-group for $s=1$;

(2) $G_{v} \cong \mathrm{A}_{4}$ or $\mathrm{S}_{4}$ for $s=2$;

(3) $G_{v} \cong \mathbb{Z}_{3} \times \mathrm{A}_{4}, \mathbb{Z}_{3} \rtimes \mathrm{S}_{4}$, or $\mathrm{S}_{3} \times \mathrm{S}_{4}$ for $s=3$.

To introduce tetravalent symmetric graphs of order $3 p$ for $p$ a prime, we define some graphs. Let $p>3$ be a prime and let $\mathbb{Z}_{3 p}=\mathbb{Z}_{3} \times \mathbb{Z}_{p}=\langle a\rangle \times\langle b\rangle$ be the cyclic group of order $3 p$. Define $\mathcal{C} A_{3 p}=\operatorname{Cay}\left(\mathbb{Z}_{3 p},\left\{a b, a^{-1} b, a b^{-1}, a^{-1} b^{-1}\right\}\right)$. By the definition of $G(3 p, 2)$ given in [23, Example 3.4], it is easy to see that $\mathcal{C} A_{3 p} \cong G(3 p, 2)$ and $\operatorname{Aut}\left(\mathcal{C} A_{3 p}\right)=\mathbb{Z}_{3 p} \rtimes \mathbb{Z}_{2}^{2}$. The next proposition is about the classification of connected tetravalent symmetric graphs of order $3 p$ (see $[23$, Theorem]).

Proposition 2.8. Let $p>7$ be a prime and $X$ a connected tetravalent symmetric graph of order $3 p$. Then $X \cong \mathcal{C} A_{3 p}$.

\section{Graph constructions and isomorphisms}

In this section we introduce connected tetravalent symmetric graphs of order $9 p$ for $p$ a prime. The first example is the lexicographic product of $C_{9}$ and $2 K_{1}$. 
Example 3.1. The lexicographic product $C_{9}\left[2 K_{1}\right]$ is defined as the graph with vertex set $V\left(C_{9}\right) \times V\left(2 K_{1}\right)$ such that for any two vertices $u=\left(x_{1}, y_{1}\right)$ and $v=\left(x_{2}, y_{2}\right)$ in $V\left(C_{9}\left[2 K_{1}\right]\right), u$ is adjacent to $v$ in $C_{9}\left[2 K_{1}\right]$ if and only if $\left\{x_{1}, x_{2}\right\} \in E\left(C_{9}\right)$. Then $C_{9}\left[2 K_{1}\right]$ is a connected tetravalent 1 -transitive Cayley graph on the group $\mathbb{Z}_{9} \times \mathbb{Z}_{2}$ and $\operatorname{Aut}\left(C_{9}\left[2 K_{1}\right]\right)=\mathbb{Z}_{2}^{9} \rtimes D_{18}$.

From [25, Example 3.2], we have the following example.

Example 3.2. Let $G=\langle a\rangle \times\langle b\rangle \times\langle c\rangle \cong \mathbb{Z}_{3} \times \mathbb{Z}_{3} \times \mathbb{Z}_{2}$. The Cayley graph $\mathcal{G}_{18}=\operatorname{Cay}\left(G,\left\{c a, c a^{-1}, c b, c b^{-1}\right\}\right)$ is 1-transitive and $\operatorname{Aut}\left(\mathcal{G}_{18}\right)=G \rtimes D_{8}$.

$\mathrm{Xu}$ and $\mathrm{Xu}[25]$ gave a classification of tetravalent arc-transitive Cayley graphs on finite abelian groups. The following example is extracted from $[25$, Example 3.2 and Theorem 3.5].

Example 3.3. Let $p \geq 3$ be a prime and $G=\langle a\rangle \times\langle b\rangle \cong \mathbb{Z}_{3} \times \mathbb{Z}_{3 p}$. Then the Cayley graph $\mathcal{C} A_{(3,3 p)}^{1}=\operatorname{Cay}\left(G,\left\{b, b^{-1}, a b, a^{-1} b^{-1}\right\}\right)$ is 1-regular and

$$
\operatorname{Aut}\left(\mathcal{C} A_{(3,3 p)}^{1}\right)=G \rtimes \mathbb{Z}_{2}^{2} \text {. }
$$

Furthermore, if $p \equiv 3(\bmod 4)$, then there is only one connected tetravalent symmetric Cayley graph on the group $G$, that is, $\mathcal{C} A_{(3,3 p)}^{1}$, and if $p \equiv 1(\bmod 4)$ there are exactly two connected tetravalent symmetric Cayley graphs on the group $G$, that is, $\mathcal{C} A_{(3,3 p)}^{1}$ and $\mathcal{C} A_{(3,3 p)}^{2}$, where $\mathcal{C} A_{(3,3 p)}^{2}=\operatorname{Cay}\left(G,\left\{b, b^{-1}, a b^{w}\right.\right.$, $\left.\left.a^{-1} b^{-w}\right\}\right)$ and $\operatorname{Aut}\left(\mathcal{C} A_{(3,3 p)}^{2}\right)=G \rtimes \mathbb{Z}_{4}$ with $w$ an element of order 4 in $\mathbb{Z}_{p}^{*}$.

By [27, Theorems 1 and 3], there is only one connected tetravalent symmetric Cayley graph on the cyclic group of order $9 p$ for each prime $p \geq 5$.

Example 3.4. Let $p \geq 5$ be a prime and $G=\langle a\rangle \times\langle b\rangle \cong \mathbb{Z}_{9} \times \mathbb{Z}_{p}$. The unique connected tetravalent symmetric Cayley graph on $G$ is $\mathcal{C} A_{9 p}=\operatorname{Cay}(G,\{a b$, $\left.\left.a^{-1} b^{-1}, a^{-1} b, a b^{-1}\right\}\right)$, which is 1-regular and its automorphism group $\operatorname{Aut}\left(\mathcal{C} A_{9 p}\right)$ $=G \rtimes \mathbb{Z}_{2}^{2}$.

Let $X=\operatorname{Cay}(H, T)$ be a connected tetravalent symmetric Cayley graph on a non-abelian group $H$ of order 27. Then $\langle T\rangle=H, T^{-1}=T$ and $|T|=$ 4. By [7, Corollary 3.2], $X$ is normal, and hence $\operatorname{Aut}(X)_{1}=\operatorname{Aut}(H, T)$ by Proposition 2.5. Since $|H|=27$, we may assume that $T=\left\{x, x^{-1}, y, y^{-1}\right\}$. Thus, $\operatorname{Aut}(H, T)$ is a 2-group and faithful on $T$, forcing that $\operatorname{Aut}(H, T) \leq D_{8}$. Since $X$ is symmetric, 4|| $\operatorname{Aut}(H, T) \mid$. By the elementary group theory, there are two non-abelian groups of order 27 :

$$
\begin{aligned}
& G_{1}(27)=\left\langle a, b \mid a^{9}=b^{3}=1, b^{-1} a b=a^{4}\right\rangle ; \\
& G_{2}(27)=\left\langle a, b, c \mid a^{3}=b^{3}=c^{3}=1,[a, b]=c,[a, c]=[b, c]=1\right\rangle .
\end{aligned}
$$

If $H=G_{1}(27)$, then $4 \nmid|\operatorname{Aut}(H)|$ because each automorphism $\alpha \in \operatorname{Aut}(H)$ has the following form:

$$
\alpha: \begin{cases}a \mapsto a^{i} b^{j}, & (i, 9)=1,0 \leq j \leq 2 \\ b \mapsto a^{3 k} b, & 0 \leq k \leq 2 .\end{cases}
$$


This is impossible because 4|| $\operatorname{Aut}(H, T) \mid$. Thus, $H=G_{2}(27)$ and $o(x)=$ $o(y)=3$, where $o(x)$ denotes the order of $x$ in $G_{2}(27)$. Since $\langle x, y\rangle=H$ and $[x, y] \in Z(H)=\langle c\rangle, a, b$ and $c$ have the same relations as do $x, y$ and $[x, y]$, which implies that the map $a \mapsto x, b \mapsto y, c \mapsto[x, y]$ induces an automorphism of $G_{2}(27)$. It follows that $X \cong \operatorname{Cay}\left(G_{2}(27), S\right)$, where $S=\left\{a, a^{-1}, b, b^{-1}\right\}$.

Clearly, the maps $a \mapsto b, b \mapsto a, c \mapsto c$ and $a \mapsto b, b \mapsto a^{-1}, c \mapsto c$ induce automorphisms of $G_{2}(27)$, say $\alpha_{1}$ and $\alpha_{2}$, respectively. Then $\alpha_{1}, \alpha_{2} \in$ Aut $\left(G_{2}(27), S\right)$ and $\left\langle\alpha_{1}, \alpha_{2}\right\rangle \cong D_{8}$, forcing that $X$ is symmetric. On the other hand, since $\operatorname{Aut}\left(G_{2}(27), S\right) \leq D_{8}$, one has that $\operatorname{Aut}\left(G_{2}(27), S\right)=D_{8}$ and $\operatorname{Aut}(X)=G_{2}(27) \rtimes D_{8}$. Thus, we have the following example.

Example 3.5. Let $G=G_{2}(27)=\langle a, b, c| a^{3}=b^{3}=c^{3}=1,[a, b]=c,[a, c]=$ $[b, c]=1\rangle$ and $S=\left\{a, a^{-1}, b, b^{-1}\right\}$. Define

$$
\mathcal{G}_{27}=\operatorname{Cay}(G, S)
$$

Then $\operatorname{Aut}\left(\mathcal{G}_{27}\right)=G \rtimes D_{8}$ and $\mathcal{G}_{27}$ is the only connected tetravalent symmetric Cayley graph on non-abelian group of order 27.

Let $X$ be a symmetric graph, and $A$ an arc-transitive subgroup of $\operatorname{Aut}(X)$. Let $\{u, v\}$ be an edge of $X$. Assume that $H=A_{u}$ is the stabilizer of $u \in V(X)$ and that $g \in A$ interchanges $u$ and $v$. It is easy to see that the core $H_{A}$ of $H$ in $A$ (the largest normal subgroup of $A$ contained in $H$ ) is trivial, and that $H g H$ consists of all elements of $A$ which maps $u$ to one of its neighbors in $X$. By $[16,20]$, the graph $X$ is isomorphic to the coset graph $\operatorname{Cos}(A, H, H g H)$, which is defined as the graph with vertex set $\{H a \mid a \in A\}$, the set of right cosets of $H$ in $A$, and edge set $\{\{H a, H d a\} \mid a \in A, d \in H g H\}$. The valency of $\operatorname{Cos}(A, H, H g H)$ is $|H g H| /|H|=\left|H: H \cap H^{g}\right|$, and $\operatorname{Cos}(A, H, H g H)$ is connected if and only if $H g H$ generates $A$. By right multiplication, every element in $A$ induces an automorphism of $\operatorname{Cos}(A, H, H g H)$. Since $H_{A}=1$, the induced action of $A$ on $V(\operatorname{Cos}(A, H, H g H))$ is faithful, and hence we may view $A$ as a group of automorphisms of $\operatorname{Cos}(A, H, H g H)$.

From [14], one can see that, up to isomorphism, there is only one primitive tetravalent symmetric graph of order $n$ if $n=45$ or 153 .

Example 3.6. Let $G=\operatorname{Aut}\left(\mathrm{A}_{6}\right) \cong \mathrm{S}_{6} \rtimes \mathbb{Z}_{2}$ and let $P$ be a Sylow 2-subgroup of $G$. By [5], $P$ is a maximal subgroup of $G$ and hence $N_{G}(P)=P$. Let $H$ be an elementary abelian 2-subgroup of $P$ of order 8 . Then $N_{G}(H) \cong \mathrm{S}_{4} \times \mathbb{Z}_{2}$. Let $d$ be an involution in $N_{G}(H) \backslash P$. Define

$$
\mathcal{G}_{45}=\operatorname{Cos}(G, P, P d P) .
$$

Then $\mathcal{G}_{45}$ is a connected tetravalent 1-transitive graph and $\operatorname{Aut}\left(\mathcal{G}_{45}\right) \cong \operatorname{Aut}\left(\mathrm{A}_{6}\right)$.

Example 3.7. Let $G=\operatorname{PSL}(2,17)$ and let $P=\langle a, b| a^{8}=b^{2}=1, b a b=$ $\left.a^{-1}\right\rangle \cong D_{16}$ be a Sylow 2-subgroup of $G$. By [5], $P$ is a maximal subgroup of $G$ and hence $N_{G}(P)=P$. Let $H=\left\langle a^{4}, b\right\rangle$. Then $N_{G}(H) \cong \mathrm{S}_{4}$. Let $d$ be an 
involution in $N_{G}(H) \backslash P$. Define

$$
\mathcal{G}_{153}=\operatorname{Cos}(G, P, P d P) .
$$

Then $\mathcal{G}_{153}$ is a connected tetravalent 1 -transitive graph and $\operatorname{Aut}\left(\mathcal{G}_{153}\right) \cong \operatorname{PSL}(2$, 17).

Since the automorphism groups of the graphs defined in Examples 3.1-3.7 are pairwise non-isomorphic, we have the following lemma.

Lemma 3.8. $C_{9}\left[2 K_{1}\right], \mathcal{G}_{18}, \mathcal{C} A_{(3,3 p)}^{1}, \mathcal{C} A_{(3,3 p)}^{2}, \mathcal{C} A_{9 p}, \mathcal{G}_{27}, \mathcal{G}_{45}$ and $\mathcal{G}_{153}$ are connected pairwise non-isomorphic tetravalent symmetric graphs.

\section{Classification}

This section is devoted to classifying tetravalent symmetric graphs of order $9 p$ for $p$ a prime. First we have the following lemma.

Lemma 4.1. Let $p$ be a prime greater than 3 and $G$ a non-abelian group of order $9 p$. Then any connected tetravalent normal Cayley graph on $G$ cannot be symmetric.

Proof. Let $X=\operatorname{Cay}(G, S)$ be a connected tetravalent normal Cayley graph. Then $\langle S\rangle=G, S^{-1}=S$ and $|S|=4$. Since $|G|=9 p$, we may assume $S=\left\{x, x^{-1}, y, y^{-1}\right\}$, and since $X$ is normal, $\operatorname{Aut}(G, S)=\operatorname{Aut}(X)_{1}$ by Proposition 2.5 .

Suppose to the contrary that $X$ is symmetric. Then $\operatorname{Aut}(G, S)$ is transitive on $S$, forcing that $o(x)=o(y)$. Note that $p>3$. By Sylow Theorem, $G$ has a normal Sylow $p$-subgroup, which means that $o(x) \neq p$ because $\langle S\rangle=G$. Denote by $Z(G)$ the center of $G$. From the elementary group theory, up to isomorphism, there are three non-abelian groups of order $9 p$ for a prime $p>3$ :

$$
\begin{aligned}
& G_{1}=\left\langle a, b \mid a^{p}=b^{9}=1, b^{-1} a b=a^{r}\right\rangle, \text { where } r \in \mathbb{Z}_{p}^{*} \text { and } o(r)=3 ; \\
& G_{2}=\left\langle a, b \mid a^{p}=b^{9}=1, b^{-1} a b=a^{s}\right\rangle, \text { where } s \in \mathbb{Z}_{p}^{*} \text { and } o(s)=9 ;
\end{aligned}
$$

$G_{3}=\left\langle a, b, c \mid a^{p}=b^{3}=c^{3}=[b, c]=[a, b]=1, c^{-1} a c=a^{t}\right\rangle$, where $t \in \mathbb{Z}_{p}^{*}$ and $o(t)=3$.

Case 1: $G=G_{1}$.

In this case, $Z(G)=\left\langle b^{3}\right\rangle$ and $Z(G)$ is the unique subgroup of order 3 in $G$. Since $\langle S\rangle=G$, one has $o(x) \neq 3$ and hence $o(x)=o(y)=3 p$ or 9. Similarly, if $o(x)=3 p$, then $G=\langle S\rangle \subseteq Z(G) \times\langle a\rangle$, a contradiction. Thus, $o(x)=9$ and $x, y$ have the form $a^{i} b^{3 j+1}$ or $a^{i} b^{3 j-1}$. Each automorphism $\alpha$ in $\operatorname{Aut}(G)$ can be written as follows:

$$
\alpha: \begin{cases}a \mapsto a^{i}, & 1 \leq i \leq p-1 \\ b \mapsto a^{j} b^{3 k+1}, & 0 \leq j \leq p-1,0 \leq k \leq 2 .\end{cases}
$$

Clearly, $\operatorname{Aut}(G)$ is transitive on the set $\left\{\left\{g, g^{-1}\right\} \mid g \in G, o(g)=9\right\}$. We may assume that $x=b$ and $y=a^{i} b^{3 k+1}$. Since $a \mapsto a^{i}, b \mapsto b$ induces an automorphism of $G, S \equiv\left\{b, b^{-1}, a b^{3 k+1},\left(a b^{3 k+1}\right)^{-1}\right\}$. Note that every automorphism 
of $G$ cannot map $b$ to $a^{i} b^{3 k-1}$. It follows that $\operatorname{Aut}(G, S) \lesssim \mathbb{Z}_{2}$. Thus, $\operatorname{Aut}(G, S)$ cannot be transitive on $S$, a contradiction.

Case 2: $G=G_{2}$.

Since $o(x) \neq p$, each element in $S$ has order 3 or 9 , and since $\left\langle a, b^{3}\right\rangle$ is a metacyclic normal subgroup of order $3 p$ containing all elements of order 3 , one has $o(x) \neq 3$. Thus, $o(x)=o(y)=9$ and $x, y$ have the form $a^{i} b^{3 j+1}$ or $a^{i} b^{3 j-1}$. Each automorphism $\alpha$ in $\operatorname{Aut}(G)$ can be written as follows:

$$
\alpha: \begin{cases}a \mapsto a^{i}, & 1 \leq i \leq p-1 \\ b \mapsto a^{j} b, & 0 \leq j \leq p-1\end{cases}
$$

Note that $a \mapsto a^{i}, b \mapsto b$ and $a \mapsto a, b^{j} \mapsto a^{k} b^{j}$ induce automorphisms of $G$. Then $S \equiv\left\{b^{3 k_{1}+1},\left(b^{3 k_{1}+1}\right)^{-1}, a b^{3 k_{2}+1},\left(a b^{3 k_{2}+1}\right)^{-1}\right\}$. Since every automorphism of $G$ cannot map $b^{i}$ to $a^{j} b^{-i}$, one has $\operatorname{Aut}(G, S) \lesssim \mathbb{Z}_{2}$. Thus, $\operatorname{Aut}(G, S)$ cannot be transitive on $S$, a contradiction.

Case 3: $G=G_{3}$.

Since $o(x) \neq p$, each element in $S$ has order $3 p$ or 3. Since $\langle a, b\rangle$ contains all elements of order $3 p$ in $G$, one has $o(x)=3$ because $\langle S\rangle=G$. Note that $Z(G)=\langle b\rangle$. Thus, $b, b^{2} \notin S$, and $x, y$ have the form $a^{i} b^{j} c$ or $a^{i} b^{j} c^{-1}$ with $1 \leq i \leq p$ and $1 \leq j \leq 3$. Each automorphism $\alpha$ in $\operatorname{Aut}(G)$ can be written as follows:

$$
\alpha: \begin{cases}a \mapsto a^{i} & 1 \leq i \leq p-1 ; \\ b \mapsto b^{j} & 1 \leq j \leq 2 \\ c \mapsto a^{k} b^{l} c & 0 \leq k \leq p-1,0 \leq l \leq 2 .\end{cases}
$$

Thus, we may assume that $x=c$, and since the map $a \mapsto a^{i}, b \mapsto b^{j}, c \mapsto c$ induces an automorphism of $G, S \equiv\left\{c, c^{-1}, a b c,(a b c)^{-1}\right\}$. Since every automorphism of $G$ cannot map $a^{i} b^{j} c$ to $\left(a^{i} b^{j} c\right)^{-1}$, one has $\operatorname{Aut}(G, S) \lesssim \mathbb{Z}_{2}$. Thus, $\operatorname{Aut}(G, S)$ cannot be transitive on $S$, a contradiction.

To state the main theorem, we introduce the so called quotient graph. Let $X$ be a graph and let $G \leq \operatorname{Aut}(X)$ be an arc-transitive subgroup on $X$. Assume that $G$ is imprimitive on $V(X)$ and $\mathcal{B}=\left\{B_{1}, B_{2}, \ldots, B_{n}\right\}$ is a complete block system of $G$. The block graph or quotient graph $X_{\mathcal{B}}$ of $X$ relative to $\mathcal{B}$ is defined as the graph with vertex set the complete block system $\mathcal{B}$, and with the two blocks adjacent if and only if there is an edge in $X$ between those two blocks. Clearly, if $X$ is $G$-symmetric, then $X_{\mathcal{B}}$ is $G / K$-symmetric, where $K$ is the kernel of $K$ on $\mathcal{B}$. For a normal subgroup $N$ of $G$, the set of the orbits of $N$ forms a complete block system of $G$. In this case we denote by $X_{N}$ the quotient graph of $X$ relative to the set of the orbits of $N$. The following is the main result of this paper.

Theorem 4.2. Let $p$ be a prime. Then any connected tetravalent symmetric graph of order $9 p$ is isomorphic to one of the graphs in Table 1. Furthermore, all graphs in Table 1 are pairwise non-isomorphic. 
TABLE 1. Tetravalent $s$-transitive graphs of order $9 p$

\begin{tabular}{|c|c|c|c|}
\hline$X$ & $s$-transitive & $\operatorname{Aut}(X)$ & Comments \\
\hline$C_{9}\left[2 K_{1}\right]$ & 1-transitive & $\mathbb{Z}_{2}^{9} \rtimes D_{18}$ & Example 3.1, $p=2$ \\
\hline $\mathcal{G}_{18}$ & 1-transitive & $\left(\mathbb{Z}_{3}^{2} \times \mathbb{Z}_{2}\right) \rtimes D_{8}$ & Example $3.2, p=2$ \\
\hline $\mathcal{G}_{27}$ & 1-transitive & $\left(\mathbb{Z}_{3}^{2} \rtimes \mathbb{Z}_{3}\right) \rtimes D_{8}$ & Example $3.5, p=3$ \\
\hline $\mathcal{G}_{45}$ & 1-transitive & $\operatorname{Aut}\left(\mathrm{A}_{6}\right)$ & Example $3.6, p=5$ \\
\hline $\mathcal{G}_{153}$ & 1-transitive & $\operatorname{PSL}(2,17)$ & Example $3.7, p=17$ \\
\hline $\mathcal{C} A_{9 p}$ & 1-regular & $\mathbb{Z}_{9 p} \rtimes \mathbb{Z}_{2}^{2}$ & Example $3.4, p \geq 5$ \\
\hline $\mathcal{C} A_{(3,3 p)}^{1}$ & 1-regular & $\left(\mathbb{Z}_{3} \times \mathbb{Z}_{3 p}\right) \rtimes \mathbb{Z}_{2}^{2}$ & Example $3.3, p \geq 3$ \\
\hline $\mathcal{C} A_{(3,3 p)}^{2}$ & 1-regular & $\left(\mathbb{Z}_{3} \times \mathbb{Z}_{3 p}\right) \rtimes \mathbb{Z}_{4}$ & Example $3.3, p \equiv 1(\bmod 4)$ \\
\hline
\end{tabular}

Proof. By Lemma 3.8, all graphs in Table 1 are connected pairwise non-isomorphic tetravalent symmetric graphs. Let $X$ be a connected tetravalent symmetric graph of order $9 p$. To finish the proof, it suffices to show that $X$ is isomorphic to one of the graphs listed in Table 1.

If $p \leq 7$, then by $[17,24]$, there are ten connected tetravalent symmetric graphs of order $9 p$ : two graphs for $p=2$, two graphs for $p=3$, four graphs for $p=5$ and two graphs for $p=7$. Thus, $X$ is isomorphic to $C_{9}\left[2 K_{2}\right], \mathcal{G}_{18}, \mathcal{G}_{27}$, $\mathcal{C} A_{(3,9)}^{1}, \mathcal{G}_{45}, \mathcal{C} A_{45}, \mathcal{C} A_{(3,15)}^{1}, \mathcal{C} A_{(3,15)}^{2}, \mathcal{C} A_{63}$ or $\mathcal{C} A_{(3,21)}^{1}$. Let $p>7$ and assume that $X$ is a normal Cayley graph. Then by Examples 3.3, 3.4 and Lemma 4.1, $X$ is isomorphic to $\mathcal{C} A_{9 p}, \mathcal{C} A_{(3,3 p)}^{1}$ or $\mathcal{C} A_{(3,3 p)}^{2}$.

Thus, in what follows one may assume that $p>7$ and $X$ is not a normal Cayley graph, that is, $A$ has no normal regular subgroup on $V(X)$. Then, to finish the proof it suffices to show that $X \cong \mathcal{G}_{153}$.

Set $A=\operatorname{Aut}(X)$ and let $A_{v}$ be the stabilizer of $v \in V(X)$ in $A$. Since $X$ is symmetric, either $A_{v}$ is a 2-group or $A_{v} \cong \mathrm{A}_{4}, \mathrm{~S}_{4}, \mathbb{Z}_{3} \times \mathrm{A}_{4}, \mathbb{Z}_{3} \rtimes \mathrm{S}_{4}$ or $\mathrm{S}_{3} \times \mathrm{S}_{4}$ by Proposition 2.7. It follows that $|A| \mid 2^{4} \cdot 3^{4} \cdot p$ or $2^{t} \cdot 3^{2} \cdot p$ for some integer $t$. Since $p>7$, every Sylow 2-subgroup of $A$ is also a Sylow 2-subgroup of a stabilizer of some vertex in $A$, implying that $A$ has no non-trivial normal 2-subgroups.

Suppose that $A$ has an intransitive minimal normal subgroup, say $N$. Since $|V(X)|=9 p$ and $|A| \mid 2^{4} \cdot 3^{4} \cdot p$ or $2^{t} \cdot 3^{2} \cdot p, N$ is either a non-abelian simple group, or an elementary abelian 3 - or $p$-group. Let $\mathcal{B}=\left\{B_{1}, B_{2}, \ldots, B_{n}\right\}$ be the set of orbits of $N$ and $K$ the kernel of $A$ acting on $\mathcal{B}$. Then $N \leq K$. Let $m=\left|B_{1}\right|$. Then $m n=9 p$ with $1<m, n<9 p$. The quotient graph $X_{N}$ has vertex set $\mathcal{B}$ and $A / K \leq \operatorname{Aut}\left(X_{N}\right)$. Moreover, assume that $B_{1}$ is adjacent to $B_{2}$ in $X_{N}$ with $v \in B_{1}$ and $u \in B_{2}$ being adjacent in $X$. Clearly, $X_{N}$ has valency 2 or 4 .

Case 1: $X_{N}$ has valency 2 .

In this case, $X_{N}$ is a cycle and $A / K \cong D_{2 n}$. Since $X$ is symmetric, the induced subgraph $\left\langle B_{1} \cup B_{2}\right\rangle$ of $B_{1} \cup B_{2}$ in $X$ is a union of several cycles of the 
same length greater than 4 , implying that $K_{v}$ is a 2-group and $K$ acts faithfully on $B_{1}$. Since $A / K \cong D_{2 n}$, one has $|A|=2^{s} m n=2^{s} 9 p$ for some integer $s$. This implies that if $A$ has a Hall $\{3, p\}$-subgroup, then it is regular on $V(X)$. Note that $m n=9 p$ with $1<m, n<9 p$. Thus, $\left\langle B_{1} \cup B_{2}\right\rangle \cong C_{2 m}, 3 C_{6}, 3 C_{2 p}$ or $p C_{6}$.

Let $\left\langle B_{1} \cup B_{2}\right\rangle \cong C_{2 m}$. Since $\operatorname{Aut}\left(C_{2 m}\right) \cong D_{4 m}$, one has $\mathbb{Z}_{m} \lesssim K \lesssim D_{2 m}$, and since $A / K \cong D_{2 n}, A$ has a normal subgroup of order $9 p$, which is regular on $V(X)$ because $A_{v}$ is a 2 -group. Thus, $A$ has a normal regular subgroup, a contradiction.

Let $\left\langle B_{1} \cup B_{2}\right\rangle \cong 3 C_{6}$. Then $N$ has blocks of length 3 on $B_{1}$ and since $K$ acts faithfully on $B_{1}, N$ must be an elementary abelian 3-group and hence $K$ is a $\{2,3\}$-group. By Proposition $2.2, K$ is solvable, and since $A / K \cong D_{2 p}$, $A$ is solvable. Thus, $A$ has a Hall $\{3, p\}$-subgroup, say $G$, which is regular on $V(X)$. Since $N \unlhd G, G$ cannot be isomorphic to $G_{1}, G_{2}$ or $G_{3}$ as listed in Lemma 4.1. It follows that $G$ is abelian, and by Proposition 2.6, $X$ is a normal Cayley graph on $G$, a contradiction.

Now let $\left\langle B_{1} \cup B_{2}\right\rangle \cong 3 C_{2 p}$ or $p C_{6}$. Then $\left|B_{1}\right|=3 p$ and since $N$ is transitive on $B_{1}, N$ must be a non-abelian simple group, say $T$. By [5, pp. 12-14], $T$ is one of the following groups in Table 2.

TABLE 2. Non-abelian simple $\{2,3, p\}$-groups extracted from [5]

\begin{tabular}{llc}
\hline Group & Order & Out \\
\hline $\mathrm{A}_{5}$ & $2^{2} \cdot 3 \cdot 5$ & 2 \\
$\mathrm{~A}_{6}$ & $2^{3} \cdot 3^{2} \cdot 5$ & $2^{2}$ \\
$\operatorname{PSL}(2,7)$ & $2^{3} \cdot 3 \cdot 7$ & 2 \\
$\operatorname{PSL}(2,8)$ & $2^{3} \cdot 3^{2} \cdot 7$ & 3 \\
$\operatorname{PSL}(2,17)$ & $2^{4} \cdot 3^{2} \cdot 17$ & 2 \\
$\operatorname{PSL}(3,3)$ & $2^{4} \cdot 3^{3} \cdot 13$ & 2 \\
$\operatorname{PSU}(3,3)$ & $2^{5} \cdot 3^{3} \cdot 7$ & 3 \\
$\operatorname{PSU}(4,2)$ & $2^{6} \cdot 3^{4} \cdot 5$ & 2 \\
\hline
\end{tabular}

If $\left\langle B_{1} \cup B_{2}\right\rangle \cong 3 C_{2 p}$, then $N$ has a transitive action of degree 3 , which is impossible because $N$ is a non-abelian simple group. Thus, $\left\langle B_{1} \cup B_{2}\right\rangle \cong p C_{6}$. Since $|A|=2^{s} m n=2^{s} 9 p$ and $N$ is intransitive, $9 p \nmid|N|$. Then by Table 2 , one has $N \cong \operatorname{PSL}(2,7)$. This is impossible because $p>7$.

Case 2: $X_{N}$ has valency 4 .

In this case, $K_{v}$ fixes the neighborhood of $v$ in $X$ pointwise. Thus, $K=N$ is semiregular on $V(X)$ and $A / N \lesssim \operatorname{Aut}\left(X_{N}\right)$. Since $|V(X)|=9 p$, one has $N=\mathbb{Z}_{p}, \mathbb{Z}_{3}^{2}$ or $\mathbb{Z}_{3}$.

Let $N \cong \mathbb{Z}_{p}$. Then the quotient graph $X_{N}$ has order 9 . By Proposition 2.4, $A / N$ contains a regular subgroup, say $B / N$, on $V\left(X_{N}\right)$, that is, $X_{N}$ is a Cayley graph on $B / N$. It follows that $|B / N|=9$ and hence $B / N$ is abelian. By 
Proposition 2.6, $B / N \unlhd A / N$ and hence $B \unlhd A$. Thus, $B$ is a normal regular subgroup of $A$ on $V(X)$, a contradiction.

Let $N \cong \mathbb{Z}_{3}^{2}$. Then $X_{N}$ is a tetravalent $A / N$-symmetric graph of order $p$. Since $p>7, X_{N}$ is not a complete graph, and hence $A / N$ has a normal regular Sylow $p$-subgroup by Proposition 2.3. This implies that $A$ has a normal regular subgroup, a contradiction.

Let $N \cong \mathbb{Z}_{3}$. Then $X_{N}$ is a connected tetravalent symmetric graph of order $3 p$. Since $p>7$, by Proposition 2.8 one has $X_{N} \cong \mathcal{C} A_{3 p}$. It follows that $A / N$ has a normal regular subgroup on $V\left(X_{N}\right)$ because $\operatorname{Aut}\left(\mathcal{C} A_{3 p}\right) \cong \mathbb{Z}_{3 p} \rtimes \mathbb{Z}_{2}^{2}$, which implies that $A$ has a normal regular subgroup on $V(X)$, a contradiction.

Now we may assume that $A$ has no intransitive minimal normal subgroup. Thus, every non-trivial normal subgroup of $A$ is transitive on $V(X)$. Again let $N$ be a minimal normal subgroup of $A$. Then $N$ is transitive on $V(X)$ and since $|V(X)|=9 p, N$ is a non-abelian simple group as listed in Table 2 . Recall that $p>7$ and either $\left|N_{v}\right|=2^{t}$ or $\left|N_{v}\right|=3 \cdot 2^{2}, 3 \cdot 2^{3}, 3^{2} \cdot 2^{2}, 3^{2} \cdot 2^{3}$ or $3^{2} \cdot 2^{4}$. It follows that $N \cong \operatorname{PSL}(2,17)$. Set $C=C_{A}(N)$, the centralizer of $N$ in $A$. Then $C \cap N=1$ and $C$ is a $\{2,3\}$-group. If $C \neq 1$, then $C$ is an intransitive normal subgroup of $A$ because $|V(X)|=9 p$, which is contrary to our assumption. Thus, $C=1$ and $A=A / C \lesssim \operatorname{Aut}(N)$ by Proposition 2.1. Since $N \cong \operatorname{PSL}(2,17)$, one has that $A=\operatorname{PSL}(2,17)$ or $\operatorname{PGL}(2,17)$, and the stabilizer $A_{v}$ is a Sylow 2-subgroup of $A$, which is maximal in $A$ by [5]. It follows that $A$ is primitive on $V(X)$, and by [14, Theorem 1.5] and Example 3.7, $X \cong \mathcal{G}_{153}$ and $A \cong \operatorname{PSL}(2,17)$.

Acknowledgements. This work was supported by the National Natural Science Foundation of China (11171020, 10961004).

\section{References}

[1] Y. G. Baik, Y.-Q. Feng, H. S. Sim, and M. Y. Xu, On the normality of Cayley graphs of abelian groups, Algebra Colloq. 5 (1998), no. 3, 297-304.

[2] N. Biggs, Algebraic Graph Theory, Second ed., Cambridge University Press, Cambridge, 1993.

[3] C. Y. Chao, On the classification of symmetric graphs with a prime number of vertices, Trans. Amer. Math. Soc. 158 (1971), 247-256.

[4] Y. Cheng and J. Oxley, On weakly symmetric graphs of order twice a prime, J. Combin. Theory B 42 (1987), no. 2, 196-211.

[5] H. J. Conway, R. T. Curtis, S. P. Norton, R. A. Parker, and R. A. Wilson, Atlas of Finite Group, Clarendon Press, Oxford, 1985.

[6] J. D. Dixon and B. Mortimer, Permutation Groups, Springer-Verlag, New York, 1996.

[7] Y.-Q. Feng and M. Y. Xu, Automorphism groups of tetravalent Cayley graphs on regular p-groups, Discrete Math. 305 (2005), no. 1-3, 354-360.

[8] A. Gardiner and C. E. Praeger, On 4-valent symmetric graphs, European J. Combin. 15 (1994), no. 4, 375-381.

[9] — A characterization of certain families of 4-valent symmetric graphs, European J. Combin. 15 (1994), no. 4, 383-397.

[10] D. Gorenstein, Finite Simple Groups, Plenum Press, New York, 1982.

[11] B. Huppert, Eudiche Gruppen I, Springer-Verlag, Berlin, 1967. 
[12] J. H. Kwak and J. M. Oh, One-regular normal Cayley graphs on dihedral groups of valency 4 or 6 with cyclic vertex stabilizer, Acta Math. Sin. (Engl. Ser.) 22 (2006), no. 5, 1305-1320.

[13] C. H. Li, On finite s-transitive graphs of odd order, J. Combin. Theory Ser. B 81 (2001), no. 2, 307-317.

[14] C. H. Li, Z. P. Lu, and D. Marušič, On primitive permutation groups with small suborbits and their orbital graphs, J. Algebra 279 (2004), no. 2, 749-770.

[15] C. H. Li, Z. P. Lu, and H. Zhang, Tetravalent edge-transitive Cayley graphs with odd number of vertices, J. Combin. Theory Ser. B 96 (2006), no. 1, 164-181.

[16] P. Lorimer, Vertex-transitive graphs: symmetric graphs of prime valency, J. Graph Theory 8 (1984), no. 1, 55-68.

[17] B. D. Mckay, Transitive graphs with fewer than twenty vertices, Math. Comp. 33 (1979), no. 147, 1101-1121.

[18] J. M. Oh and K. W. Hwang, Construction of one-regular graphs of valency 4 and 6, Discrete Math. 278 (2004), no. 1-3, 195-207.

[19] D. J. Robinson, A Course in the Theory of Groups, Springer-Verlag, New York, 1982.

[20] B. O. Sabidussi, Vertex-transitive graphs, Monatsh. Math. 68 (1964), 426-438.

[21] C. Q. Wang and M. Y. Xu, Non-normal one-regular and 4-valent Cayley graphs of dihedral groups $D_{2 n}$, European J. Combin. 27 (2006), no. 5, 750-766.

[22] C. Q. Wang and Z. Y. Zhou, 4-valent one-regular normal Cayley graphs of dihedral groups, Acta Math. Sin. Chin. Ser. 49 (2006), 669-678.

[23] R. J. Wang and M. Y. Xu, A classification of symmetric graphs of order $3 p$, J. Combin. Theory Ser. B 58 (1993), no. 2, 197-216.

[24] S. Wilson and P. Potočnik, A census of edge-transitive tetravalent graphs, http:// jan.ucc.nau.edu/ swilson/C4Site/index.html.

[25] J. Xu and M. Y. Xu, Arc-transitive Cayley graphs of valency at most four on abelian groups, Southeast Asian Bull. Math. 25 (2001), no. 2, 355-363.

[26] M. Y. Xu, Automorphism groups and isomorphisms of Cayley digraphs, Discrete Math. 182 (1998), no. 1-3, 309-319.

[27] _ A note on one-regular graphs of valency 4, Chinese Science Bull. 45 (2000), $2160-2162$.

[28] - A note on permutation groups and their regular subgroups, J. Aust. Math. Soc. 85 (2008), no. 2, 283-287.

[29] J.-X. Zhou, Tetravalent s-transitive graphs of order 4p, Discrete Math. 309 (2009), no. 20, 6081-6086.

[30] J.-X. Zhou and Y.-Q. Feng, Tetravalent s-transitive graphs of order twice a prime power, J. Aust. Math. Soc. 88 (2010), no. 2, 277-288.

[31] — , Tetravalent one-regular graphs of order 2pq, J. Algebraic Combin. 29 (2009), no. $4,457-471$

SONG-TAO GuO

Department of Mathematics

BeiJing JiAotong University

Beijing 100044, P. R. China

E-mail address: gsongtao@gmail.com

YAN-QUAN FENG

Department of Mathematics

BeiJing Jiaotong University

Beijing 100044, P. R. China

E-mail address: yqfeng@bjtu.edu.cn 